\title{
Evaluation of Major Factors Affecting Spatial Resolution of Gamma-Rays Camera
}

\author{
Hongwei Xie*, Jianhua Zhang, Jinchuan Chen, Faqiang Zhang, Linbo Li, Jianming Qi, Yanyun Chu \\ Institute of Nuclear Physics and Chemistry, China Academy of Engineering Physic, Mianyang, China. \\ Email: *xiehw1966@sina.com
}

Received October $10^{\text {th }}, 2013$; revised November $10^{\text {th }}, 2013$; accepted November $11^{\text {th }}, 2013$

Copyright (C) 2013 Hongwei Xie et al. This is an open access article distributed under the Creative Commons Attribution License, which permits unrestricted use, distribution, and reproduction in any medium, provided the original work is properly cited.

\begin{abstract}
The spatial resolution of the gamma-rays camera was measured on a ${ }^{60} \mathrm{Co}$ gamma-rays source with edge method. The gamma-rays camera is consisting with rays-fluorescence convertor, optical imaging system, MCP image intensifier, CCD camera, electronic control system and other devices, and is mainly used in the image diagnostics of the intense pulse radiation sources [1]. Due to the relatively big quantum detective efficiency (DQE) and quantum gain of the gamma-rays, etc., the experimental data were processed by averaging multiple images and fitting curves. According to the experimental results, the spatial resolution MTF (modulation transfer function) at the $10 \%$ intensity was about 2 $\mathrm{lp} / \mathrm{mm}$. Meanwhile, because of the relatively big dispersion effects of the fluorescence transmissions in the scintillator and the optical imaging system, the maximal single-noise ratio (SNR) of the camera was found to be about 5:1. In addition, the spatial resolution of the camera was measured with pulse X-rays with $0.3 \mathrm{MeV}$ in average energy and exclusion of the effects of secondary electrons from consideration. Accordingly, the spatial resolution MTF at the 10\% intensity was about $5 \mathrm{lp} / \mathrm{mm}$. This could be an additional evidence to verify the effects of secondary electrons induced by the $1.25 \mathrm{MeV}$ gamma-rays in the scintillator upon the spatial resolution. Based on our analysis, the dispersion sizes of the secondary electrons in the scintillator are about $0.4 \mathrm{~mm}-0.6 \mathrm{~mm}$. Comparatively, as indicated by the detailed analysis of the spatial resolutions of the MCP image intensifier and CCD devices, both of them have little effect on the spatial resolution of the gamma-rays camera that could be well neglected.
\end{abstract}

Keywords: Gamma-Rays Camera; Point Spread Function (PSF); Gamma-Rays Image Detector; Scintillator; MCP Image Intensifier; CCD

\section{Introduction}

A $\gamma$-rays camera was developed for the image diagnostics of the intense pulsed $\gamma$-rays radiation sources, which was consisting with rays-fluorescence convertor, optical imaging system, MCP + CCD, electronic control system and other devices. Due to its good performances in various tunable (adjustable) parameters including exposure time spot, exposure time duration and gain, etc., the camera has been widely used in the framing image diagnostics of the pulse radiation fields $[1,2]$.

The point spread function (PSF) of the image diagnostic system is not only the major technical specification of image system performances, but also a major factor to cause the image degradation. For a given or a certain PSF, it could be considered as a major criteria for the uncer-

${ }^{*}$ Corresponding author. tainty evaluation of a given image and for the image super-resolution reconstruction. Other parameters to describe the system spatial resolution are including modulation transfer function (MTF) and the linear spread function (LSF), etc. The integration of PSF along the 1-D direction would then provide the LSF, which would then provide MTF after the Furrier Transform. All of three parameters (PSF, LSF and MTF) could reflect the spatial resolution of the rays system [3].

The edge method is a common method for the measurement of spatial resolution of $\gamma$-rays cameras [3]. In its application, the edge would be placed in a $2^{\circ}-3^{\circ}$ with the pixel array to minimize the effects of the sub-pixels upon the spatial resolution, and the data would be fitted to the curves to proved a very smooth measurement data at the same time. However, in dealing with the spatial resolution measurement of the $\gamma$-rays cameras with thick 
scintillator and/or high energy, such a data processing might be technically difficult in various aspects as follows [4]: 1) the big DQE fluctuations induced by the interactions between the high energy $\gamma$-rays and scintillator; 2) the relatively big quantum gain of the $\gamma$-raysfluorescence convertor; 3 ) the relatively more measurement steps of the system. Another important factor to affect the spatial resolution is the measurement condition. Presumably, an ideal parallel radiation source with step distribution would be most desirable for the measurement. However, the measurement of the spatial resolution would inevitably affected by various factors, including the size of radiation source, the system structure and the working conditions of system, etc. Moreover, the data process from edge spread function (ESF) to LSF and then to LSF would also amplify the noise. All of the factors add up to lead a relatively big uncertainty in the spatial resolution of the high energy $\gamma$-rays cameras. In order to contain the DQE effects upon the measurement results, serial procedures are applied as follows. Firstly, multiple ESFs would be averaged. Then, the averaged ESF would then be subject to linear fitting to provide a relatively uniform and smooth ESF. And the LSF would be obtained after the integration of the ESF. Finally, the MTF and PSF could be well available after a certain process.

The geometrical spatial resolution of the $\gamma$-rays camera is about $0.1 \mathrm{~mm} / \mathrm{pixel}$. And in order to provide a higher $\mathrm{DQE}$, some special methods were introduced, e.g. to use the optical structure with short distance and big field of view and to increase the scintillator, etc. The spatial resolution of the $\gamma$-rays camera was measured on a Co radiation source with edge method. At the same time, the spatial resolutions of the camera components were studied respectively for the scintillator, MCP image intensifier and CCD camera, etc., to provide some valuable reference for the further improvement of the spatial resolution of the $\gamma$-rays camera.

\section{Experimental Set-Ups}

\subsection{Gamma-Rays Camera}

The $\gamma$-rays camera was developed by Institute of Nuclear Physics and Chemistry, China Academy of Engineering Physic (INPC, CAEP) for scientific experiments. Characterized in high sensitivity, high resolution and big dynamic range, the camera is mainly used in the image diagnostics of the high energy $\gamma$-rays sources. The limit field of view of the camera is $\Phi 100 \mathrm{~mm}$. The process could roughly be interpreted as follows. Firstly, the radiation source is imaged onto the imaging plane by the $\gamma$-rays. Then the fluorescence image is imaged onto the incident plane of the MCP image intensifier through the optical imaging system and conversion system. Finally, the intensified image is recorded by CCD device, and the
MCP and CCD is coupled with fiber plate. Thus, the major tow parts of the system are $\gamma$-rays imagines system and the image recording system. During the process, a 10 mm YAG crystal is used to convert the $\gamma$-rays into the fluorescence image. Besides, a copper reflector with an efficiency of over $95 \%$ is placed in a $45^{\circ}$ angle with $\gamma$ rays direction to reflect the fluorescence image. Another purpose of the reflector is to avoid the direct $\gamma$-rays irradiation onto the MCP image intensifier and CCD camera. The specially developed imaging system could provide an amplification factor of 5:1 and a light collection efficiency of over $95 \%$. The MCP image intensifier was manufactured by Proxitronic Co. Ltd with a spatial resolution of over $37 \mathrm{lp} / \mathrm{mm}$. Besides, the intensifier is also characterized in tunable shutter time and electron gain, which could facilitate the imaging of the $\gamma$-rays at different time. Finally, the amplification factor of the fiber plate used to couple the MCP and CCD is 1.5:1, providing a stable and reliable recording system. And the CCD camera used in the experiment was provided by Andor Co. Ltd specifically for scientific purpose, with a pixel array of $1024 \times 1024$, pixel size of $13.3 \mu \mathrm{m} \times 13.3 \mu \mathrm{m}$, and a digital data output in 16 bit. Moreover, the CCD could be used in a very low temperature even below $-65^{\circ} \mathrm{C}$, making it a favorite option for the imaging diagnostic with low signal intensity. The total system is placed into an iron container to provide a sealed and shielded environment to avoid the effects of the electromagnetic effects upon the measurement result.

\subsection{Spatial Resolution Measurement with Edge Method}

The experiment was carried out on the intense Co source in CAEP. The schematic experimental set-up is given in Figure 1. The $60 \mathrm{Co} \gamma$-rays source used in the experiment is a line source with a geometrical parameter of $\Phi$

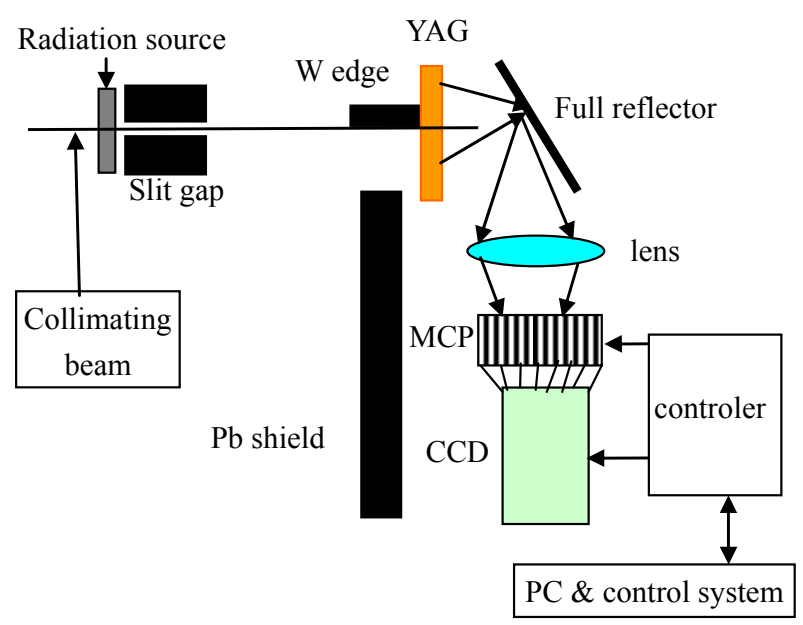

Figure 1. The schematic diagram for the PSF measurement of the $\gamma$-rays camera. 
$11 \mathrm{~mm} \times 450 \mathrm{~mm}$. And the source intensity for every line is about $10^{4} \mathrm{Ci}\left(1 \mathrm{Ci}=3.7 \times 10^{14} \mathrm{~Bq}\right)$. A $5 \mathrm{~mm} \times 50 \mathrm{~mm}$ $\mathrm{Pb}$ silt gap (200 $\mathrm{mm}$ in thickness) was used for the beam collimation and constraint. A $100 \mathrm{~mm}$ tungsten edge collimator was placed in front of but closely contacted with scintillator at the same time. The radiation flux of the $\gamma$-rays at the scintillator was about $9.0 \times 10^{6} \gamma / \mathrm{cm}^{2} \cdot \mathrm{s}$. As for the system collimation and adjustment, the procedures might be described as follows. Firstly, a laser beam $0.5 \mathrm{~mm}$ in diameter was transmitted to transverse the front center and back center of the slit gap collimator. Then the beam would be irradiated onto the scintillator surface perpendicularly. Finally, the $100 \mathrm{~mm}$-thick tungsten edge would be placed parallel with the laser beam, and the edge image would be adjusted to be at the center of the field of view of the system. In addition, due to high sensitivity of the MCP image intensifier and CCD devices to the $\gamma$-rays, another $10 \mathrm{~cm}$-thick $\mathrm{Pb}$ shield was used to protect the system. After installation and adjustment, the radiographic system could be operated with PC remote control.

\section{Experimental Data and Data Processing}

\subsection{Performance Calibration of Radiographic System}

Firstly, the flat field response is a major standard to evaluate the response uniformity of the radiographic system. A Pb collimating aperture (100 $\mathrm{mm}$ in diameter) was used to provide beam constraint and shield for the radiation source, and a YAG scintillator was placed 400 $\mathrm{cm}$ away from the radiation source. With such a set up, the $\gamma$-rays could presumably have a homogenous spatial distribution. The image signals corresponding to various radiation doses were experimentally calibrated. According to the results, the flat field responses under various radiation doses were same, the intensities in the central region were relatively high, and the non-homogeneities in the central region and edge region were about $30 \%$. The flat field images are mainly used for the homogenous correction of the radiographic images. After the correction, the images could convey information about the intensity distribution of the incident $\gamma$-rays.

Secondly, the geometrical distortion of the radiographic system was also calibrated with black-and-white squares $(5 \mathrm{~mm} \times 5 \mathrm{~mm}$ in size). The image signals were obtained and analyzed with the image processing programs. As indicated by the results, the geometrical distortion of the radiographic system was in agreement with the experimental requirements.

Finally, the dynamic sensitivity of the radiographic system was calibrated. The homogenous $\gamma$-rays were used to irradiate the YAG scintillator, and the radiation images corresponding to various radiation doses were recorded. After data processing, the relationship curves between the radiation doses and image signal intensities could be well obtained. As indicated by the curves, with a radiation dose rage of $1.0 \times 10^{6}-2.0 \times 10^{8} \gamma \cdot \mathrm{cm}^{-2}$, the radiographic system could demonstrate a fairly good linearity.

\subsection{Experimental Data}

The edge image experimentally obtained is given in Figure 2. Despite of the fact that a $100 \mathrm{~mm}$-thick W collimator was placed in region with relatively lower intensity to provide an attenuation (a decay) factor of more than $10^{-6}$, the SNR of the system was found to be only $5: 1$. The major reason for this might be attributed to the fluorescence dispersion effects through out the fluorescence transmittance, including: 1) the dispersion in the scintillator; 2) the dispersion in the optical system; 3 ) the dispersion in the MCP image intensifier; 4) the dispersions in the coupling fiber plate between the MCP and $\mathrm{CCD}$ and the ambient materials. Despite of the Pb shield of more than $10 \mathrm{~cm}$ in thickness, the scattered $\gamma$-rays along the optical path still acted as a non-negligible source to induce the noise.

Due to the relatively big non-homogeneity of the system, only an effective region $100 \times 100$ in size was adopted for data processing. As shown in Figure 2, the image signals fluctuated in relatively big amplitude with a standard deviation of about 3753 . The reasons for this might be attributed to the single-particle detecting effects of the scintillator and the relatively high quantum gains of the scintillator and MCP image intensifier, etc. Thus, during the data processing, the average was made for 100-line data. After that, the averaged data were subject to Gaussian fitting. Finally, the ESF curves could be obtained to be very uniform and smooth (as shown in Figure 3).

\subsection{Spatial Distribution of System}

Both of the MTF and PSF are important parameters to evaluate the spatial resolution of the radiographic system, but very difficult to be measured directly. However, they are functions of the LSF. LSF could be determined by

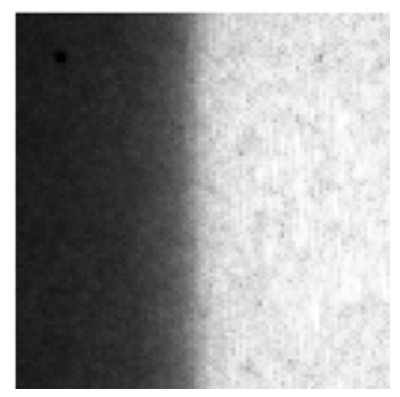

Figure 2. The experimentally obtained edge fluorescence image. 


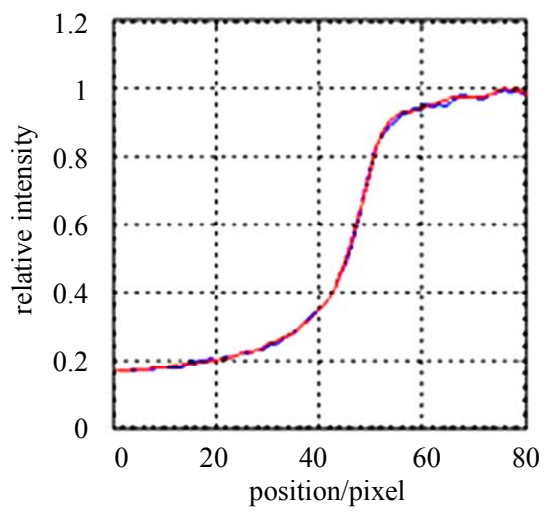

Figure 3. The experimentally obtained ESF distribution.

differentiating ESF. And the relationships among LSF, MTF and PSF could be described in the following equations. For a given ESF, the LSF could be determined in Equation (1) [4]:

$$
\operatorname{LSF}(x)=\frac{d[E S F(x)]}{d x}
$$

Since MTF is the result of the Furrier Transform of LSF, we have:

$$
\operatorname{MTF}(f)=\left|\frac{\int_{-\infty}^{\infty} L S F(x) \exp (-j 2 \pi f x) d x}{\int_{-\infty}^{\infty} L S F(x) d x}\right|
$$

And the relationship between PSF and LSF could be given as follows: [4]

$$
\operatorname{PSF}(r)=-\frac{1}{\pi} \int_{r}^{\infty} \frac{\{d[L S F(x)] / d x\}}{\left(x^{2}-r^{2}\right)^{\frac{1}{2}}} d x
$$

Based on the equations mentioned above, after processing of the edge image, the LSF, MTF and PSF of the $\gamma$-rays camera could be obtained as given in Figure 4. As shown in the figure, with $10 \%$ intensity, the MTF is only $2 \mathrm{lp} / \mathrm{mm}$, and the point spread size is about $2 \mathrm{~mm}$.

\section{Major Factors Affecting Spatial Resolution}

The spatial resolution of the $\gamma$-rays camera was measured with edge method. In the following parts, the effects of YAG scintillator, MCP image intensifier and CCD camera upon the system spatial resolution will be analyzed, respectively.

\subsection{PSF of CCD Device}

The spatial resolution of CCD device is mainly dependent on the pixel size and the electron diffusion in the silica substrate. In the silica substrate, the electron-hole pairs would be induced by photons. And the electrons would spread in the substrate and move driven by the superposed voltage before stored in the potential wells. The spread size or dimension of the electrons in the silica substrate is dependent on the superposed voltage $[5,6]$. And the superposed voltage is then dependent on the substrate thickness and manufacturing process. With a superposed voltage ranging in $2.84 \mathrm{~V}-115.4 \mathrm{~V}$, the electron spread sizes are within $48.8 \mu \mathrm{m}$ to $3.7 \mu \mathrm{m}$, and the electron spread function is basically in accordance with the Gaussian function distribution. The LSF of the electrons could be described in the following equation [6]:

$$
L S F(x)=a+b \cdot \exp \left(-\frac{\left(x-x_{0}\right)^{2}}{2 \sigma^{2}}\right)
$$

where: $\mathrm{a}, \mathrm{b}, \mathrm{x}_{0}$ and $\sigma$ are fitting constants. The spread of the electrons in the silica substrate could be obtained by using a point light source with a negligible size, subpixel sampling method and Gaussian function fitting [7]. In the high precision image measurement, the electron

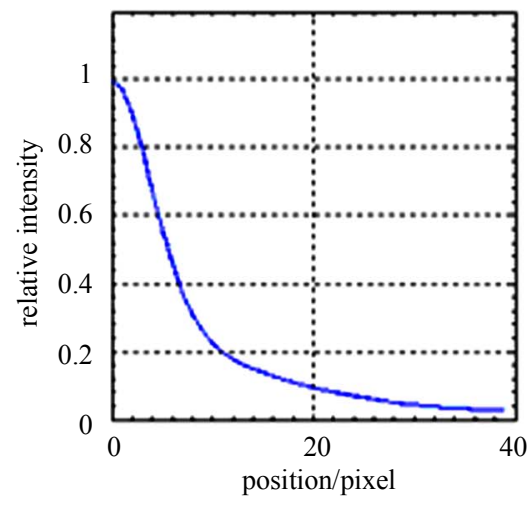

LSF
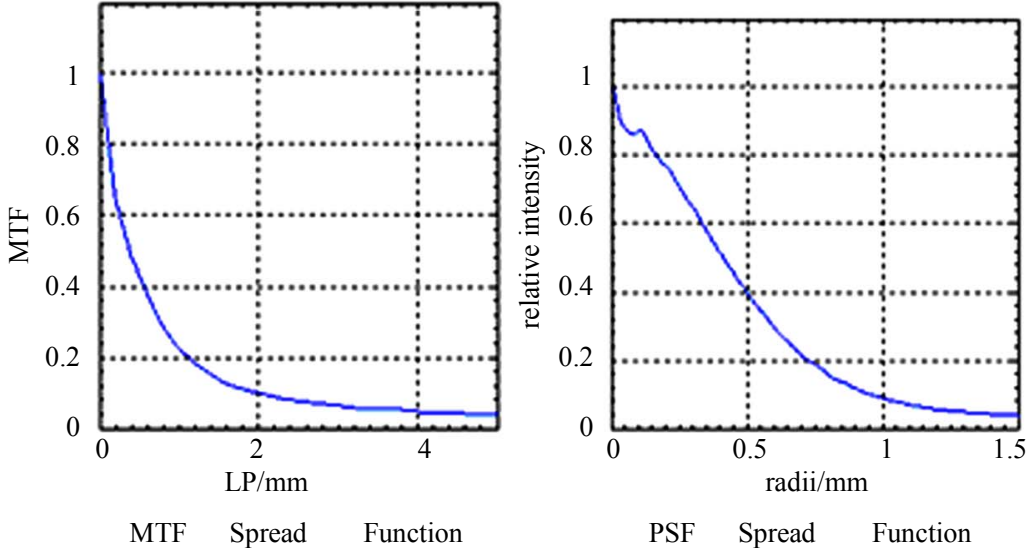

PSF Spread Function

Figure 4. The experimentally obtained LSF, MTF and PSF of the $\gamma$-rays camera. 
spread in the silica substrate is a non-negligible factor to cause the degradation. Thus, in the common design of the scientific-level high sensitivity CCD, the back illumination method is used, and the thickness of the silica substrate is deliberately determined to be $10 \mu \mathrm{m}-15 \mu \mathrm{m}$, which is roughly equal to the electron spread sizes $(5-10$ $\mu \mathrm{m})$ [7]. The typical electron PSF is given in Figure 5. As shown in the figure, the electron spread size is about $20 \mu \mathrm{m}$ corresponding to $10 \%$ intensity. Specifically, for a CCD with a pixel size of $13.3 \mu \mathrm{m} \times 13.3 \mu \mathrm{m}$, the electron spread might be about 1 to 2 pixels.

\subsection{PSF of MCP Image Intensifier}

The MCP image intensifier is mainly consisting with optical cathode, micro-channel plate (MCP) and fluorescence screen. The MCP location is between the optical cathode and fluorescence screen. The MCP input plane is $0.1 \mathrm{~mm}-0.3 \mathrm{~mm}$ away from the optical cathode and the output plane is about $0.5 \mathrm{~mm}-1.3 \mathrm{~mm}$ away from the screen. Firstly, the photoelectrons are excited from the cathode by the incident beams. Then, driven by forward accelerating electric field between the cathode and MCP input plane, the electrons would be injected into the MCP input plane almost along a straight direction before being multiplied in the micro-channels. Then the electrons would be transmitted out of the MCP output plane and accelerated again by the electric field between the output plane and the screen. Finally, the electrons would impact onto the screen to generate the corresponding fluorescence image.

The spatial resolution of the MCP image intensifier is dependent on multiple factors, including various superposed voltages, the exposure quantity of the optoelectronic cathode and the manufacturing process, etc. Besides, the dispersion effect of the electrons in the cathode and fluorescence screen would be another important factor to damage the spatial resolution. The MCP image intensifier used in the experiment could provide a spatial resolution of more than $37 \mathrm{lp} / \mathrm{mm}$. And the PSF could be given as follows [8]:

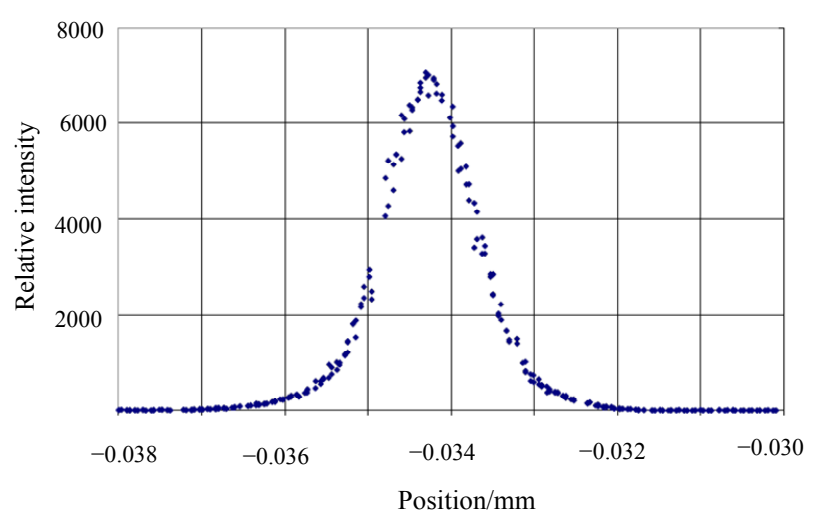

Figure 5. The spread size of electrons in the silica substrate.

$$
P S F(r)=\exp \left[-(r / 0.02)^{2}\right]
$$

where: $r$ stands for the radius (mm). The electron spread function of a typical MCP image intensifier is given in Figure 6. As shown in the figure, the diameter is about $0.06 \mathrm{~mm}$ corresponding to $10 \%$ of PSF. Take account the minification factor of 1.5 , and the spatial resolution of the MCP image intensifier is basically equal to that of the CCD camera.

\subsection{Fluorescence Dispersion Effects}

The fluorescence dispersion could be divided into two parts. One is the fluorescence dispersion in the scintillator; another one is due to the defocusing in the optical imaging system. The secondary electrons would be generated by the interactions between the $\gamma$-rays and the scintillator. And the electrons would move within the scintillator and induce energy deposition. Part of this energy would be converted into fluorescent lights. Each of the electron trajectories would be acting as a point light source. Due to the homogenous spatial distribution of the lights, only some of the lights could be collected by the light collecting system, while most of them would be dispersed within the scintillator. During the dispersion, in the interface, half penetration half refection and full reflection occur, while in the scintillator, only scattering takes place. The deliberately designed optical imaging system used in the experiment was made up of 12 lenses to provide a light collecting efficiency $30^{\circ}$. At the same time, the defocusing effects induced by the fluorescence dispersion would also affect the spatial resolution.

Similarly, the edge method was again used to measure the fluorescence spatial resolution in the radiographic system. The spatial resolution of the fluorescence was calibrated on a pulsed X-rays source with energy of about $0.3 \mathrm{MeV}$. The distance between the YAG scintillator with the radiation source was about $250 \mathrm{~cm}$, and the spatial resolution of X-rays was presumably homoge-

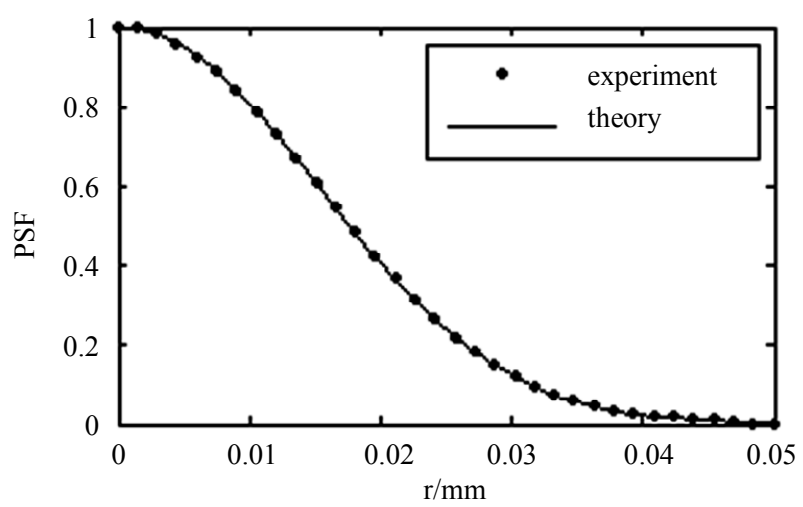

Figure 6. The experimentally obtained electron spread function of the MCP image intensifier. 
nous. In addition, the effects of transmittance range the secondary electrons was neglected, which had been rationalized experimentally and theoretically. During the experiment, a $0.1 \mathrm{~mm}$-thick copper film was tightly contacted with the scintillator surface, and the film was reasonably considered as the edge in the experiment. The SNR of the optical imaging system was finally found to be 10:1. After data processing, the MTF of the spatial resolution corresponding to $10 \%$ intensity was about 5 $\mathrm{lp} / \mathrm{mm}$ (as shown in Figure 7), which was obtained with the additional contributions from the effects if the spatial resolutions of the MCP image intensifier and CCD device upon the fluorescence transmission.

\subsection{Transmittance Range of Secondary Electrons in Scintillator}

The spatial resolution of the $\gamma$-rays in the YAG crystal is mainly dependent on the transmittance range of the secondary electrons and the fluorescence dispersion in the scintillator, etc. $[9,10]$. The interactions between the $\gamma$ rays and the scintillator would generate secondary electrons. Each of the electrons would be a small light source, whose size would be decisive for the resolution limit. In order to get the fluorescence size of a single secondary electron, an optical imaging system with high light collecting efficiency was adopted in addition to a high sensitivity camera consisting with a MCP image intensifier and cooler-equipped CCD camera. And the movement trajectories of the secondary electrons were experimentally obtained.

The experiment was carried out on a Co radiation source, whose principles are same with those given in Figure 1. The distance between the scintillator and the radiation source was $400 \mathrm{~cm}$, and the radiation dose on the scintillator was $3.7 \times 10^{7} \mathrm{~cm}^{-2} \cdot \mathrm{s}^{-1}$. At the same time, a shading plate $30 \mathrm{~mm}$ in diameter was place behind the scintillator to contain the effective image size within this range. The exposure time was $0.1 \mathrm{~ms}$. The fluorescence image is given in Figure 8. As shown in the figure, there're miscellaneous speckle effects.
As for the miscellaneous speckle effects in the image, the speckle images are in array distribution, and there's nearly no signs for the linear propagation of the electrons. The reason for this might be attributed to the limit of the spatial resolution of the optical system and the fluorescence dispersion effects in the scintillator and transmission system. Due to their effects, only the degraded electron trajectories could be observed in the image.

With energy higher than $0.1 \mathrm{MeV}$, the ratios between the energy depositions in the scintillator with the luminescent efficiencies could be considered to be equal. In this extent, for any of the speckle arrays, approximately, the signal intensities over 50\% higher than the peak would be classified as the electron trajectories, while those below those level would be the results of the scattered fluorescence. According to the estimation of the miscellaneous speckle sizes, the electron dispersion size is predictably 2 to 3 pixles, which, in dealing with the PSF of about 4 to 6 pixels, corresponds to a spread size of $0.4 \mathrm{~mm}$ to $0.6 \mathrm{~mm}$ size.

For a detailed description of the PSF of the $\gamma$-rays in the scintillator, MCNP program was introduced to track and simulate the transportation process of the $\gamma$-rays in the scintillator. And for the theoretical computation, the electronic fluorescence efficiency was supposed to be proportional to the electronic energy deposition efficiency. Other conditions for the computation were including the scintillator thickness $(10 \mathrm{~mm})$ and the interval of the radiuses of the concentric cylinders $(0.010 \mathrm{~mm})$. The energy disposition distributions in the scitillator were calculated for the $\gamma$-rays respectively $0.3 \mathrm{MeV}$ and 1.25 $\mathrm{MeV}$ in energy, whose results are given in Figure 9. As shown in the figure, the maximal transmittance range of the $0.3 \mathrm{MeV} \gamma$-rays on the projecting plane is about 0.2 $\mathrm{mm}$, while the range increases to be $0.8 \mathrm{~mm}$ for 1.25 $\mathrm{MeV}$ rays. And the PSF sizes corresponding to the $1 \%$ intensity are $0.44 \mathrm{~mm}$.

\section{Conclusion}

The spatial resolution of the $\gamma$-rays camera was measured

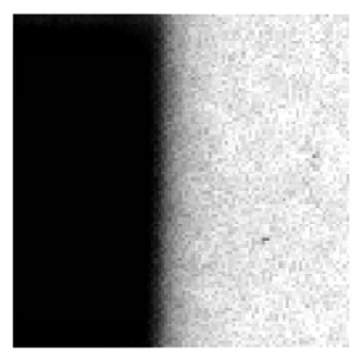

The knife image

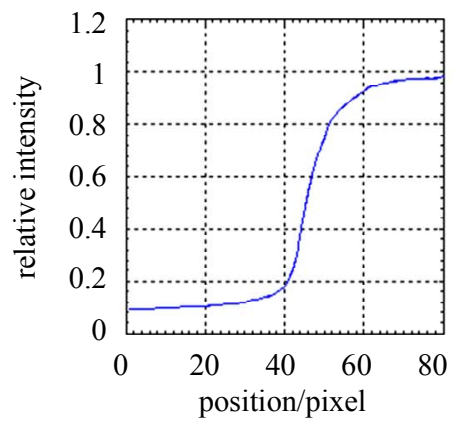

The edge spread function

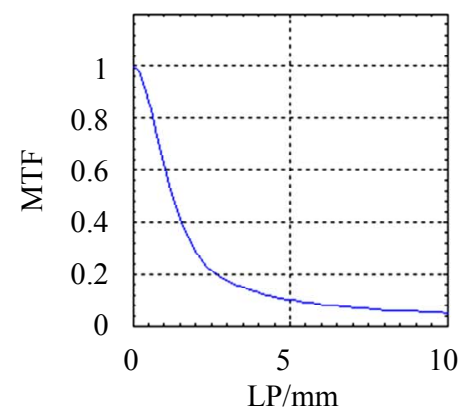

MTF

Figure 7. The spatial resolution of the fluorescence transmission in the system. 


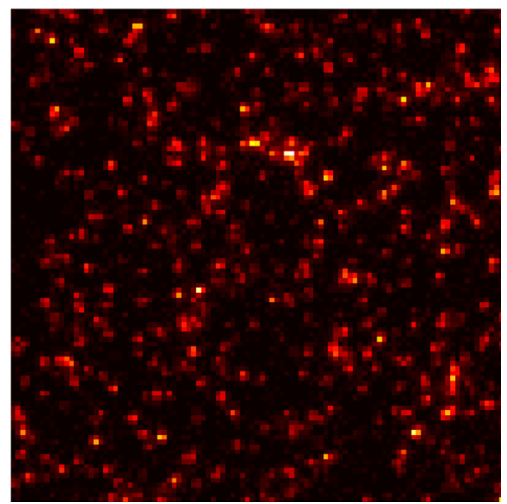

No.: 2010B0103006 and 2011B0103017) and National Natural Science Foundation (Contract No.11005095 and No.11305155). And the experimental study was made on the pulsed X-rays source in Northwest Institute of Nuclear Technology and with great help from Prof. Guo Jianming. The authors owe great gratitude to all of them.

\section{REFERENCES}

[1] S. Watson, T. Kauppila, et al., "The High-Energy Radiographic Machine Emitting X-Rays Flash Radiographic Camera," SPIE Proceedings, Vol. 2869, 1997, pp. 920928.

Figure 8. The speckle effects of the secondary electrons in the scintillator.

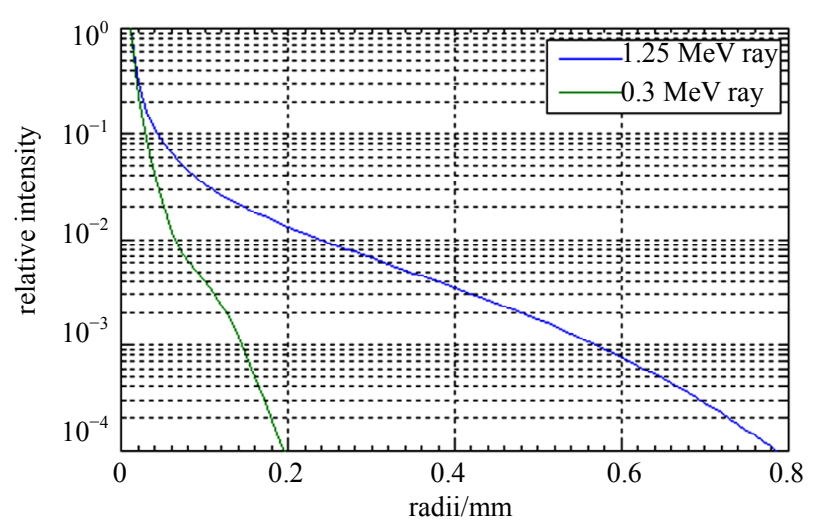

[2] G. J. Yates and N. S. P. King, "High-Frame Intensified Fast Optically Shuttered TV Cameras with Selected Imaging Applications," SPIE Proceedings, Vol. 2273, 1997, pp. 126-149.

[3] P. B. Gree and T. van Doorn, "Evaluation of an Algorithm for the Assessment of the MTF Using an Edge Method," Medical Physics, Vol. 27, No. 9, 2000, pp. 20482059.

[4] H. Q. Zhu, "Study on the Image Restoration of Y-Ray Pinhole Imaging System," Ph.D. Thesis, Tsinghua University, Beijing, 2008.

[5] P. Z. Takacs and I. Kotov, "PSF and MTF Measurement Methods for Thick CCD Sensor Characterization," SPIE Proceedings, Vol. 7742, 20120, Article ID: 774207.

[6] A. Karcher and C. J. Bebek, "Measurement of Lateral Charge Diffusion in Thick Fully Depleted, Back-Illuminated CCDs," IEEE Transactions on Nuclear Science, Vol. 51, No. 5, 2004, pp. 2231-2237. http://dx.doi.org/10.1109/TNS.2004.834721

on a $1.25 \mathrm{MeV}$ source with edge method. Due to the relatively big quantum detective efficiency (DQE) and quantum gain of the gamma-rays, etc., the experimental data were processed by averaging multiple images and fitting curves. According to the experimental results, the MTF (modulation transfer function) of spatial resolution at the $10 \%$ intensity was about $2 \mathrm{lp} / \mathrm{mm}$. Based on this, further analysis was made for the spatial resolutions of the MCP image intensifier and CCD device. Besides, the spatial resolution of the scintillating fluorescence in the $\gamma$-rays camera was measured as well as the dispersion effects of the secondary electrons in the scintillator. As indicated by the results, both the fluorescence dispersion and the dispersion of the secondary electrons are the major factors that affect the spatial resolution.

\section{Acknowledgements}

The study presented in this paper is sponsored jointly by CAEP Sci. \& Tech. Development Foundation (Contract

[7] H. Schwarzer and A. Boerner, "Dynamic PSF and MTF Measurement on a 9k TDI CCD," SPIE Proceedings, Vol. 7106, 2008. http://dx.doi.org/10.1117/12.797055

[8] H. Q. Zhu and K. L. Wang, "MTF Measurement and Analysis of Microchannel Plate Image Intensifiers," Acta Photonica Sinica, Vol. 36, No. 11, 2007, pp. 1983-1986.

[9] J. M. Ma and K. L. Wang, "Research on PSF of Scintillator as $\gamma$-Ray Fluorescenc Image Converter," Nuclear Electronics \& Detection Technology, Vol. 31, No. 4, 2011, pp. 473-478.

[10] J. H. Zhu and S. L. Niu, "Energy Deposition of Gamma Rays in LSO Crystal," High Power Laser and Particle Beams, Vol. 22, No. 6, 2010, pp. 1351-1354. 